\title{
Matrogimnasia y el desarrollo motor en niños entre 7 y 8 años con parálisis cerebral Matrogymnasia and motor development in children between 7 and 8 years old with cerebral palsy \\ *Luis Patricio Jami Vargas, *Nancy Elisabeth Caisapanta Acaro, **Ruth Narcisa Zambrano Pintado, **Diego Mauricio Bonillajurado \\ *Unidad EducativaEspecializadaC arlos Garbay (Ecuador), **UniversidadTecnológicalndoamérica(Ecuador)
}

Resumen. El siguiente estudio tiene por objetivo, mejorar el desarrollo motor grueso en niños desiete años de edad, con parálisis cerebral infantil (PCI), despuésdeunaintervención del método conocido como laM atrogimnasia. Basado en unametodologíadonde incluyen alos padresy representantes del menor, paramezclar la parte afectivay al mismo tiempo, reforzar losejerciciosen el hogar. Esunainvestigación detipo mixta, descriptiva, fenomenológica, de campo, apoyadoshermenéuticamente en lasteoríasquepermita aclarar y soportar lainvestigación. El estudio estábasado en larelevanciadelosejerciciosfisioterapéuticosque permiteen primer lugar, desarrollar el sistemamotor de losniños con PCl. Segundo, incluir en el método alos padresy representantes, con el propósito de intensificar el afecto familiar, y reforzar losejerciciosen el seno del hogar.Y, en tercer lugar, demostrar laimportanciadelaM atrogimnasia como método parael mejoramiento en niñoscon $\mathrm{PCl}$. Se proponelaadecuación de unaseriedeejercicios bajo supervisión profesional especializada, queayudeal desarrollo cognitivo y motriz delosmenorescon laintención demejorar su condición en cuanto alasposturas corporalesymentales.

Palabrasclave: adaptación instrumento FuncionesM otorasG ruesas, afecto familiar, desar rollo motriz, Matrogimnasia, motricidad gruesa, parálisiscerebral infantil, terapiafísica.

Abstract. The following study aimsto improvegross motor skill development in children seven year sof old, with cerebral palsy, after an intervention of themethod known as M atrogymnasia. Based on amethodology that includesthe parents and representatives of the minor, to mix the affective part and at the sametime, reinforcetheexercises at home. It isamixed, descriptive, phenomenological, field research, hermeneutically supported in theories that al low to clarify and support-research. The study is based on the relevance of physiotherapy exercises that allows, first, to develop themotor system of children with children cerebral palsy. Second, include in the method the parentsand representatives of the minor, with theintention of intensifying family affection and reinforce exercises from home. And thirdly, demonstrate the importance of M atrogymnasia as amethod for improvement in children with children cerebral palsy. It isproposed the adaptation of aseries of exercises under specialized professional supervision, which helpsthecognitive and motor development of minors with theintention of improving their condition in terms of bodily and mental postures.

Keywords: adaptation G ross M otor Function M easure, children cerebral palsy, family affection, grossmotor skills, Matrogymnasia, motor development, physical therapy.

\section{Introducción}

La importancia, el descubrimiento y la comprensión de los procesos de la evolución en general, vienen siendo uno de los alcances más sobresalientes en la historia de la ciencia (Cortés, 2003). Esta evolución explica de manera clara y sencilla las diferentes formas de vida en la tierra. Siendo muy corroborada en repetidas oportunidades por medio de la observación y la experimentación en un amplio espectro de disciplinas científicas (U ribe, 2017).

La ciencia evolutiva permite sentar bases sólidas, permitiendo abrir las puertas a diferentes investigacio-

Fecha recepción: 17-08-20. Fecha de aceptación: 09-12-20 Diego Mauricio Bonilla Jurado diegobonilla@uti.edu.ec nes, conduciendo al descubrimiento y desarrollo de técnicas que pueden ayudar a la prevención y combatir las diferentes enfermedades existentes ( 0 rganización de Estados Iberoamericanos para la Educación, la Ciencia y la Cultura [OEI], 2012). Por todo esto, se deben introducir conceptos tantos científicos, como no científicos, sobrelaevolución de lacienciaen las aulas para incentivar a los estudiantes y a los mismos profesionales de la enseñanza, a realzarla de manera científica.

Para M oreno \& Rivas (2017); Solís (1994), la evolución de la ciencia proporciona un ejemplo excelente de cómo trabajan los científicos. Su enfoque se encuentra en la observación de la natural eza, formulando preguntas que ponen a prueba el mundo natural y lo que quieren descubrir. Por supuesto, quetodas esasinterrogantes deben pasar por un proceso de evaluación, mediante experimentos y nuevas observaciones hasta lograr ob- 
tener resultados satisfactorios que impulsen avances modernos, significativos y científicos.

Con relación a lo anterior, la siguiente investigación trata acerca de la M atrogimnasia término que se manifiesta en Alemania a partir de las investigaciones realizadas por Hermann O nhesorge y que Helmut Schutz (citados en Pinzón, 2015) retoma trayendo un estudio que servía para la ejercitación entre madre e hijo, proponiendo ejercicios gimnásticos y actividades recreativas, que son válidas realizarlas en la escuela y en el hogar por su facilidad de aplicación y bajo costo en la utilización de implementos o materiales. La palabra Matrogimnasia proviene del griego que quiere decir en sus raíces «matro», madre y «gimnasia» relacionado con el ejercicio.

ParaJami (2019) la M atrogimnasiaes conocida como el conjunto de tareas físicas que logran integrar vínculos-físicosy afectivos- entreel grupo familiar y terapista, es decir, existe una combinación de estímulos donde fisioterapista, padre e hijos interactúan con el propósito de fortalecer mediante la implementación de ejercicios especializados y focal izados para obtener resultados motrices que cambian actitudes, roles y funciones del niño para adquirir confianza y seguridad propia. En este mismo sentido, Joaquín (2014) expone que la Matrogimnasia es un programa pedagógico secuencial que tiene vinculación estrecha entre padres, madres 0 cuidadores, en este caso con los hijos. Bajo este progra ma pedagógico se desea indicar el acercamiento importante de los involucrados para el fortalecimiento del espacio socio afectivo, corporal y psicomotriz.

Cabe destacar lo que resalta Anangonó (2016); Pinos, Ayala \& Bonilla (2018); Pacheco (2015) respecto a la participación que tiene la familia en los programas que a lo largo del desarrollo de los niños van corrigiendo, donde cada miembro debe tomar una serie de decisiones y canalizar distintas tareas diarias que influyen de manera decisiva en el rendimiento de los menores, siendo 0 catalogado el seno familiar el espacio ideal donde se debe reforzar cada uno de los ejercicios necesarios para la evolución. Por otro lado, de esta forma se va creando un nexo importante entre los integrantes del núcleo familiar, convirtiéndose los padres en el motor principal para lograr ese desarrollo corporal, mental, motriz y emocional del menor. De todo esto se desprende, la importancia que tiene la familia durante el proceso de la Matrogimnasia, donde los profesionales practicantes, deben involucrar a todo el entorno del menor, para la obtención de resultados satisfactorios y evolutivos.
La M atrogimnasia en la actual idad resulta inusual y, más aún combinada en niños de siete años que padecen de parálisis cerebral infantil (PCI), por ende, el objetivo de esta investigación, es explicar la importancia de contribuir con un programa de actividad física que permita mejorar las habilidades motoras gruesa, vistas como: su control cefálico, sedente, gateo, bipedestación y marchaen niños con $\mathrm{PCl}$. Al respecto expresan López, M oreno \& López (2020) que mediante la actividad física se consiguen resultados positivos, ya que la misma, es considerada una estrategia que traza beneficios en la salud y promueven el desarrollo motor de los niños. Afianzan también, que es considerada como una herra mienta para desarrollar aspectos personales sobre todo en el área motor y social.

En otro orden de ideas, la parálisis cerebral infantil (PCI) es una expresión más común para describir a menores con problemas neurológicos posturales y del movimiento motriz (Solórzano \& Vera, 2018; Jadán et al., 2020; Eliasson, Krumlindem, Rösblad, Beckung, Arner, 0 hrvall \& Rosenbaum, 2006). Dichos problemas neurológicos comienzan a aparecer durante los primeros años de vida y, generalmente no empeoran con el tiempo. DestacaVázquez \& Vidal (2014) que el diagnostico siempre involucra su déficit en desarrollo motor, especial retraso en sus habilidades motoras gruesas, y otros síntomas de disfunción cerebral y trastornos sensoriales.

Gómez, Jaimes, Palencia, Hernández \& Guerrero (2013) argumentan que la PCl es un síndrome que se encuentra localizado en el sistema nervioso central (SNC), en la primera neurona o también conocidacomo neurona motora superior. Esto implica que generalmente casi todos los niños que presentan $\mathrm{PCl}$, deben tener presente los defectos en cuanto a la postura y al movimiento, además de otrostrastornos asociados. Ante esto, se afirma que la $\mathrm{PCl}$ es una de las causas más frecuente, relacionada con la discapacidad motriz en la edad pediátrica.

Para Lorente \& Bugie (1988); Wollack (2004) la PCI o encefalopatía estática, constituyen una serie de trastornos motrices que producen serias anomalías de la postura, así como del tono muscular y la coordinación motora. Todo esto, debido a la probabilidad de una lesión congénita que puede afectar directamente al cerebro inmaduro de los niños, e inclusive, pudiera ser de naturaleza no progresiva, persistente, estática y una con tendencia a mejorar o permanecer toda la vida.

Por todo lo anterior, se puede afirmar que la $\mathrm{PCl}$ es considerada como un trastorno del desarrollo del tono 
postural y del movimiento de carácter persistente, condicionando las actividades motrices en sus actividades cotidianas, sin descuidar a una agresión menos progresiva a un cerebro inmaduro, en este caso del niño. Para Bonilla, Pérez, Jami \& Camacho (2019) los procesos evolutivos relacionados con el desarrollo cognitivo, es efectivo en los primeros años de vida, aunque durante la evolución psicológica sea bastante independiente de las características físicas. Estos desarrollos vienen acompañados de otros factores agregados, tales como: sensitivos, cognitivos, lenguaje, perceptivos, conducta, epilepsia, musculo-esqueléticos; cuya existencia o no, puede condicionar de forma significativa el pronóstico individual inicial de los niños.

Las diferentes edades, tienen mucha importancia respecto a la PCI, y en este sentido, Lyon (1990) hace una clasificación de los tipos clínicos (figura 1).

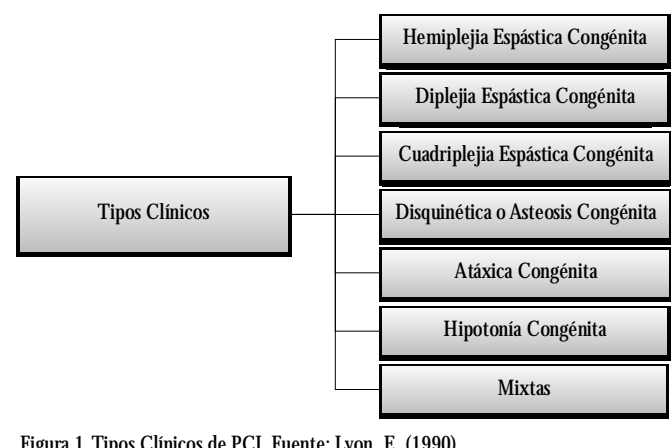

\section{H emiplejia Espástica Congénita}

Afectación motora unilateral, habitualmente de tipo espástico, congénita si ocurre antes del final del período neonatal y adquirida si ocurre después. Es asintomática duranteel período neonatal, con intervalos silentes hasta los seis meses. Es más común en varones y en el lado derecho del cuerpo, destacando signos notorios motores que suelen representar el constante uso de unamano para alcanzar objetos que le llamen la atención.

\section{Diplejia Espástica Congénita}

Altera la parte motora bilateral conjuntamente con los miembros inferiores, donde se ven afectados directamente los miembros superiores. Es la forma más común de $\mathrm{PCl}$, convergiendo entre dos formas de presentación: diplejia espástica y atáxica. En cuanto a la primera, el signo más sobresaliente es el aumento del tono muscular en las extremidades inferiores.

\section{Cuadriplejia Espástica Congénita}

Es una de las más graves y su principal característica es la espasticidad bilateral predominante en los miem- bros superiores. Así mismo se destaca, la ausencia del habla y las alteraciones perceptivo-sensoriales, falta de apetito y problemas pulmonares de manera más acentuados.

\section{D isquinética o Atetosis Congénita}

Los primeros síntomas pudieran aparecer a los cinco 0 diez meses, seguido de movimientos pocos flexibles de miembros. Lo recomendable es esperar hasta los dos años para poder hacer una eval uación clínicareal. Generalmente se altera el tono axial, interfiriendo la sedestación y bipedestación.

\section{Atáxica Congénita}

Conocida como ataxia cerebelosa no progresiva. Puede no empezar hasta después del primer o segundo año de edad, aunque no se descar tala presenciahipotonía en la época de lactancia y retraso madurativo motor. Los primeros síntomas suelen ser oscilación del tronco para intentar mantener la sedestación.

\section{H ipotonía Congénita}

Es poco frecuente y los lactantes presentan debilidades en las piernas. Se presenta retrasos atenuantes en el desarrollo motriz y la existencia de reflejos tendinosos profundosehiperactivos. U na manera de visualizar ciertos síntomas, es cuando los niños se sujetan por debajo de los brazos, estos flexionan ambas piernas por las caderas.

\section{Mixtas}

Presentan signos y síntomas espásticos y extrapiramidales. Se afecta directamente las partes motoras, trayendo como consecuencia, compromisos en zonas encefálicas, con secuelas de deterioro de ganglios basales, corteza y región subcortical.

Por esto, el presente estudio demuestra la importancia que tiene la M atrogimnasia en el desarrollo motor en niños de siete años con $\mathrm{PCl}$. Al respecto, N ovoa (1991) presenta una clasificación interesante acerca de la $\mathrm{PCl}$ desde el punto de vista nosológico, que va en función de: sitio anatómico, etiología, clínica, topogra fía, fisiopatología, terapéutica clase A-B-C y D, extensión de la afectación funcional, trastornos asociados y la neuro-imagen.

La investigación se basa en la clasificación de tipo terapéutica de case $D$, tomando en consideración su significado para el estudio, el cual va dirigido al ingreso en una institución de rehabilitación por tiempo prolonga do, con la ayuda de especialistas, médicos, terapeutas, 
padres y representantes; que deben realizar la consecución de los ejercicios, en este caso, matrogimnásticos para poder elevar el desarrollo motriz de los niños.

Para ir cerrando el tema de la $\mathrm{PCl}$, es pertinente incluir un mensaje del trabajo de Low (1987), el cual expresaque $~_{\ldots . .}$. estos niños no pueden ser curados, pero pueden ser ayudados estableciendo objetivos terapéuticos realistas y específicos» (p.91). Por lo tanto, la importancia que reviste la M atrogimnasia, se refleja mediante la adaptación de ejercicios motrices que ayuden al desarrollo motriz de los niños en especial con PCl. Además, agrega el autor: «... esta ayuda sería más efectiva cuando todos los que proporcionan cuidado y trata miento tengan el interés y el potencial de cada niño en sus corazones y en sus mentes» (p.92). Esto refuerza el interés que deba poner el entorno familiar, los nexos afectivos, el apego que deben recibir para que el trata miento arroje resultados positivos y satisfactorios.

Tomando en consideración el objetivo principal de este estudio, el cual es mejorar el desarrollo motor grueso en niños con $\mathrm{PCl}$ después de una intervención de la Matrogimnasia, es notorio resaltar cuales serían los posibles ejercicios que se podrían adaptar, con el propósito de formar niños más seguros de sí mismos, presentando un análisis cognitivo con capacidad hacia la toma de decisiones, bajo responsabilidades propias de la edad con inteligencia emocional, permitiéndoles aceptarse a sí mismo y a los demás, siguiendo una correla ción social con los padres y otros adultos. Es básicamente investigar el entorno que en la Matrogimnasia existe, para proponer ejercicios que ayude al menor objeto de estudio.

Bajo estas perspectivas, Campistol, Arroyo, Póo \& Ruggieri (2011); Póo \& Campistol (2007), hacen énfa sis en el apoyo fisioterapéutico, resaltando el control postural y del movimiento siempre de mayor a menor grado, destacando que los niños con tratamiento de $\mathrm{PCI}$ deben incluir una serie de ejercicios bajo supervisión profesional especializada. Por su parte y, en este mismo orden de ideas, Robaina, Riesgo \& Robaina (2013) proponen el método Bobath, el cual es uno de los más conocidos y empleados en la U nión Europea en lasúltimas décadas. Este método es utilizado especialmente en niños con $\mathrm{PCl}$, y su éxito se centra en la cooperación y participación de los padres para coadyuvar a desar rollar su máximo potencial. La importancia de la participa ción de los padres durante y después de la sesión de tratamiento es vital, ya que permite observar y aprender aspectos relacionados con el control postural, el manejo en el vestir, actividades de juego, alimentación, entre otros.

0 tro método que se destaca, es el expuesto por Alvarado, Cervantes, Carrasco \& García (2015) Ilamado el método Vojta. Basado específicamente en la estimulación de determinados reflejos posturales complejos, como instrumento para la obtención de secuencias motores coordinados. La estimulación se utiliza como propioceptivas, provocando al mismo tiempo, la coordinación de movimientos del decúbito ventral.

El método Peto, propuesto por López (2015) también conocido como la educación conductiva, va dirigido hacia la rehabilitación integral con la intención fundamental que el menor obtenga un mayor grado de independencia posible. Para cubrir el objetivo de la investigación, llama mucho laatención del método Bobath debido a la importancia que tiene la familia como centro motor del éxito que también puede tener la Matrogimnasia en los niños con $\mathrm{PCl}$. Sin embargo, independientemente del método utilizado, es fundamental la colaboración general de todas las personas que rodean el entorno de los niños para obtener el desarrollo motriz esperado.

\section{Material y método}

\section{Diseño y participante}

La investigación es de tipo iterativo o mixto, también conocidos como integrativa, multi-métodos, métodos múltiples, estudios de triangulación (Hernández, M éndez, M endoza\& Cueva, 2017; Creswell \& Creswell, 2018; Lieber \& Weisner, 2010). A lo que HernándezSampieri \& M endoza (2018) exponen:

Los métodos mixtos representan un conjunto de procesos sistemáticos, empíricos y críticos de investigación e implican la recolección y el análisis de datos cuantitativos y cualitativos, así como su integración y discusión conjunta, para realizar inferencias producto de toda la información recabada (metainferencias) y logran un mayor entendimiento del fenómeno bajo estudio. (p. 612)

Además, existe la combinación de estrategias etnográficas de análisis social, con el propósito de diseñar de una teoría fundamentada basada en Strauss $\&$ Corbin (2002) a diseñar una investigación-acción y, tener bases para confirmar y demostrar a la M atrogimnasia como una metodología. La variable independiente es medida antes y después de la manipulación de las varia bles. Se emplea al grupo de sujetos de control y experimental, empleando un estudio prospectivo longitudinal en un determinado tiempo. 
El tipo de muestra para la encuesta es de 16 niños con el rango de siete años de edad y para el pre y posttest fueron de diez, empleando lo queM ejía(2010) plantea en cuanto a dos criterios de referencia. El primero de inclusión, en niños de cinco años con diagnóstico médico de $\mathrm{PCI}$ confirmado y la aceptación de un acompañante para avalar el proceso evaluativo. El segundo, deexclusión, conformados por niños con patología o trastornos integrados que obstaculicen la implementación adecuada del instrumento; y la presencia de elementos contextuales que pongan en riesgo la condición de salud del niño durante la participación en el estudio.

La muestra pertenece a la U nidad Educativa Especializada Carlos Garbay, situada en la provincia de Chimborazo, ciudad Riobamba, Ecuador, para ellos se aplicó el muestreo no probabilístico, basado en Hernández-Sampieri, 0 p. Cit., debido a que la selección de la misma va orientada por las características y contexto del estudio y no por un criterio generalizado, es decir, que la finalidad no es la generalización probabilísticos más bien la multiplicidad de los propósitos de la investigación.

La edad comprendida de la muestra estudiada será entre siete y ocho años de edad, los niños serán asignados a dos grupos experimentales y uno de control para obtener mejores resultados cuasi-experimentales -equipos ya predeterminados- al exponer los instrumentos. Cabe destacar que, para obtener resultados satisfactorios, los primeros recibirán la Matrogimnasia y el otro grupo no se le aplicará el programa.

\section{Instrumentos}

El instrumento principal de la investigación es el Gross M otor Function M easure (GMFM) adaptación de Mejía, O p. Cit., que viene asociado de herramientas metodológicas como la verificación y supervisión, para consolidar la efectividad y eficiencia del proceso de la Matrogimnasia que responda a la atención de los niños con $\mathrm{PCl}$. Por lo tanto, aplicar de manera sistemática, acorde, correcta y coherente el MGFM; permitió el fortalecimiento del desarrollo motor y el entorno socio emocional de los menores. Dicho instrumento se aplicó antes y después de aplicar la M atrogimnasia para medir los avances y la fiabilidad, obteniendo un índice de validez de contenido mayor de 0,8 cifra pertinente y acorde al lenguaje para los niños evaluados.

Lo resaltante e importante en cuanto a la veracidad de los resultados, viene enmarcado en la minimización de los sesgos al momento de emplear el MGFM, neutralizando la información para que sea totalmente real y acorde a las situaciones presentadas en los niños con $\mathrm{PCl}$. La determinación de seleccionar la muestra de forma aleatoria, permite afianzar consistentemente el instrumento el cual es adaptado a las diferentes condiciones socio-culturales de los menores.

El MGFM sirvió de guía de diligenciamiento donde su efectividad se afianza en el entrenamiento adecuado para los fisioterapeutas evaluadores, para mantenerse en concordancia con los datos que se quieren recopilar, y dar veracidad que dichos resultados concuerden con lo observado y verificado cumpliendo con el formato de evaluación practicado.

\section{Procedimiento}

El procedimiento se organiza por fases de cumplimiento, para evitar olvidar cualquier detalle en la implementación del instrumento y obtener los resultados suficientes para concluir de manera eficiente sobre el tema estudiado. Las fases se muestran a continuación en la tabla 1.

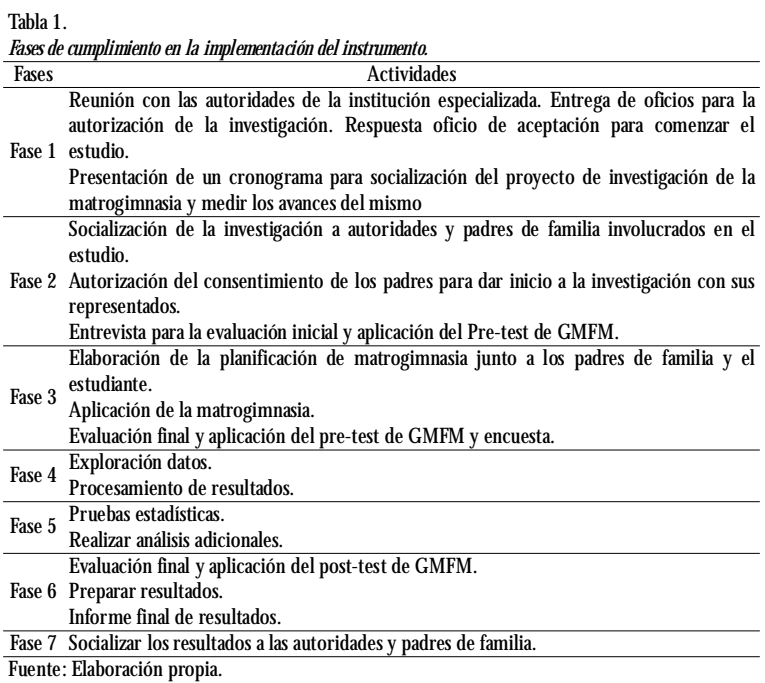

\section{Análisis de datos}

Para esta investigación se aplicó la prueba estandarizada llamada test de medida de la función motora, Gross M otor Function M easure (GMFM), con adaptación de contenido lingüístico, como apoyo parala atención del desarrollo motor en estudiantes con parálisis cerebral, donde se revisarán la información de los datos del pre-test y post-test para analizar las distribuciones de variables y procedemos e interpretar pruebas estadísticas para la probar la hipótesis planteada.

La importancia radica en la toma de resultados antes y después de practicar la herramienta de la M atrogimnasia y poder evaluar los resultados de los niños y la efectividad del proceso. Los avances en este sentido, sustentan las conclusiones de la investigación. 


\section{Resultados}

Los resultados arrojados en la investigación, permitieron hacer análisis y observaciones que afianzan la importancia que tiene la M atrogimnasia para el desarrollo motriz en los niños de siete años de edad con $\mathrm{PCl}$, tomando en consideración la participación de los padres, representantes y responsables de reforzar los ejercicios en casa.

Cuando se consultó acerca del conocimiento de la importancia de la Matrogimnasia conjunta en los pa dres, se constató que dichos representantes tienen presente lo importante que resulta para sus hijos reforzar la implementación de estos ejercicios matrogimnásicos en el hogar, es decir, que el $100 \%$ de los consultados lo corroboran. Lo relevante de conocer lo que significa y representan los ejercicios de manera efectivida, produce que se intensifican la participación adicional. Cuando realmente se logra internalizar, es necesario la ayuda 0 reforzamiento de los ejercicios para que los resultados sean más rápidos y evidentes, es donde se comienzan observar cambios relevantes, dicho de otra manera, cuando forman parte (fisioterapista, padres, representantes y paciente) del cumpliendo del programa de M atrogimnasia de forma adecuada y disciplinada desde el hogar, se puede tener una recuperación satisfactoria y efectiva a mediano plazo.

En relación al grado de participación de los ejercicios desde el hogar, se relevó la falta de interés existente en la participación de los padres y representantes al respecto. El $44 \%$ de los encuestados claramente expresan que sólo se quedan con lo suministrado en la U nidad Educativa Especializada. Es importante resaltar, que reforzar los ejercicios desde el hogar produce efectos marcados y efectivos. Es necesario reforzar lo mencionado anteriormente sobre tener pleno conocimiento de la utilidad e importancia de la Matrogimnasia en niños con PCl. La importancia del compartir tiempo necesario para la prosecución del desarrollo del menor, es necesario. Sin embargo, al consultar a los padres y representantes encargados de los ejercicios de los niños el $31 \%$ declara que no tienen tiempo para dedicarles a sus hijos al gunas horas de la semana para el compartir. Llama mucho la atención de este porcentaje, porque estos niños de siete años con PCI necesitan más atención de lo normal. El tiempo debe ser fundamental para poder organizarse y emplear de forma disciplinada los ejercicios de reforzamiento que agilizarían a mediano plazo, el desarrollo del niño. Recordemos que la integración y el afecto que se debe fomentar, ayuda de ma nera significativa al menor.

Uno de los resultados más relevantes, surge desde el apoyo que se le pueden brindan a los niños con $\mathrm{PCl}$, ya que puede venir desde varios puntos de vistas, y uno de ellos es el estímulo hacia el deporte. Sin embargo, el $13 \%$ de los encuestados respondieron que no incentivan a realizar ninguna actividad física de sus hijos extra curriculares, lo que claramente se entiende como un descuido total una vez que el niño llega al hogar. El deporte y cualquier actividad extra es una herramienta no solo de control motriz sino que a su vez permite que el niño desarrolle otrostipos de sentimiento. Jugar, montar bicicleta, bailar, la alegría, la risa, quemar energía, ilusionarse por parecerse a al gún deportista, permite desarrollar patrones que sirven para activar procesos motrices que estimulan al niño.

0 tro punto de vista importante que se destaca dentro de los resultados de la investigación, es el tema del apoyo familiar. Este acercamiento resulta primordial en el proceso de la Matrogimnasia, pero de manera inesperada, al momento de ser encuestados en este sentido, se pudo determinar que el $19 \%$ de los padres y representante no disponen de tiempo suficiente debido a diferentes motivos que están por encima de la situación de su hijo. Es importante empezar a realizar campañas que ayuden a entender a los padres y representante la importancia de seguir en cualquier entorno el apoyo hacia su hijo. Lo relevante es no quedarse sola mente con lo practicado en el Centro de Especializa ción. La integración, disciplina y dedicación serán esenciales durante el proceso de la Matrogimnasia.

Un resultado interesante el cual debe ser importante atacar, fue cuando se le consultó por orden de importancia de menor a mayor siendo 1 el menos importante y 5 el más importante, la forma de for tal ecer los vínculos afectivos dentro del núcleo familiar, arrojando los siguientes resultados: (1) mediante las comidas, (2) reforzando losejerciciosmatrogimnásicos, (3) no hay tiempo, (4) no hay espacio hacia el vínculo afectivo y (5) realizando actividades juntos. Una vez más se ratifica, que el reforzar los ejercicios referentes a la Matrogimnasia es bajo desde el hogar. Sólo se les practica con regularidad si asisten a la U nidad Educativa Especializada. Hay que recordarles a los padres y representantes, que mientras se hagan los refuerzos en el hogar, los resultados serán más notorios.

Cuando se realiza una investigación, resulta suma mente necesario revisar todos los ángulos y perspectivas existentes, para poder tomar los correctivos necesarios y dar cumplimiento de los ciclos. Al consultar si 
el centro de Unidad Educativa Especializada «Carlos Garbay» fomenta el refuerzo de los ejercicios matrogimnásticos en el hogar, la respuesta no fue la más esperada, ya que el $56 \%$ de los encuestados dijo que sí. El refuerzo más importante debe venir de los profesionales que lo proporcionan, además, deben explicar cómo se hacen y para qué sirven.

Los beneficios positivos de la Matrogimnasia deben ser dados a conocer por parte del equipo de profesionales. La falta de información arroja que el $31 \%$ de los encuestados desconocen el beneficio del mismo. Deben existir programas que sean dirigidos a los padres y representantes, para que conozcan de cerca el programa y lo beneficioso que resulta su adecuada implementación en niños de siete años con PCl. Los Centros de Enseñanzas deben buscar el mecanismo ideal para poder llevar la información necesaria para que los representantes entiendan el proceso.

A continuación, se presentan los principales datos generales, así como los principales resultados encontra dos durante el levantamiento de información realizado en la Unidad Educativa Especializada «Carlos Garbay» ciudad Riobamba, provincia de Chimborazo. Respecto al género de los niños que inter vienen en la recolección de la información, nueve son masculinos y una es femenina. La edad comprendida de los menores es de siete años. La raza que rigen es la siguiente: nueve mestizos y una indígena, y final mente, entre los tipos de lesiones en relación a los niños con discapacidad física motora en la Unidad Educativa se tiene que dos son leves, uno moderada y siete con lesión de diagnóstica.

Los resultados al momento de aplicar el instrumento entre los grupos (control y experimental) que intervienen en la investigación, son los siguientes:
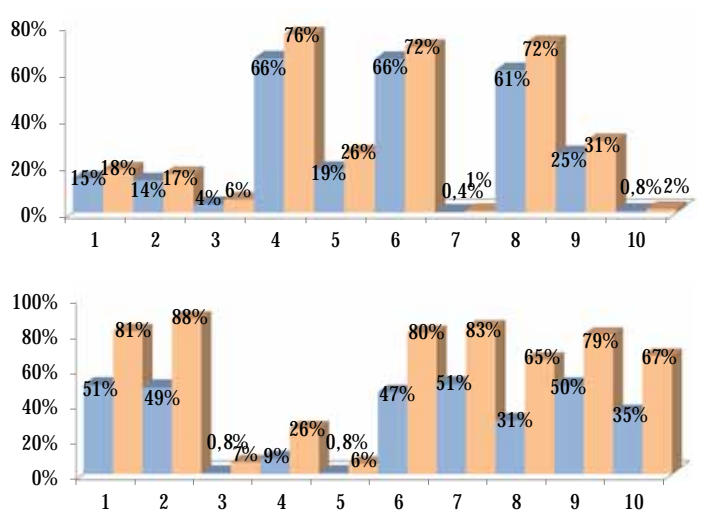

Figura 2. Resultados finales pre-test vs post-test

Como se muestra en la figura 2, los resultados de la primera evaluación del test GMFM , tanto para el grupo de control como para el grupo experimental, eviden- cian porcentajes que no superan el $67 \%$ de habilidades motoras gruesas en los mejores evaluados. Para la segunda evaluación -post test- del GMFM, se evidencia una mejora sustancial para la gran mayoría de casos de los dos grupos evaluados, donde se supera el $88 \%$ de habilidades motoras gruesas como es el caso del niño dos del grupo experimental, posterior ala aplicación de técnicas de Matrogimnasia. En el caso del grupo experimental, el $100 \%$ de los participantes muestran mejoría en sus habilidades motoras gruesas después del empleo de la Matrogimnasia.

Seguidamente, se detallan los resultados obtenidos de las dimensiones del Test GMFM -decúbitos y rotaciones, sentados, gateo y posición de rodillas, bipedestación, marcha-.

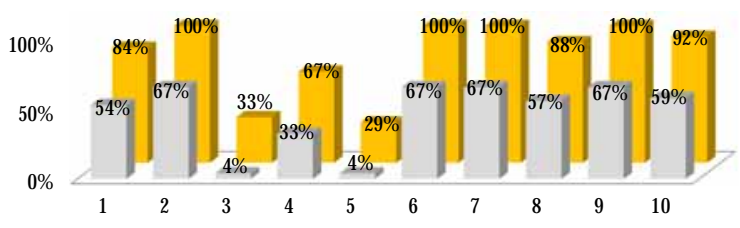

Figura 3. Resultados finales - Dimensión cúbitos y rotaciones

De la totalidad de mejores evaluados con el Test GMFM, el $100 \%$ de la población infantil presenta una mejoría en la primera dimensión del test. Los niños con menor desarrollo de esta dimensión después de la eva luación post-test son los niños 3 y 5 con porcentajes de $33 \%$ y $29 \%$ respectivamente. Los niños con mayor porcentaje de desarrollo de esta área son los infantes dos, seis, siete y nueve ( $100 \%$ de desarrollo en esta dimensión).

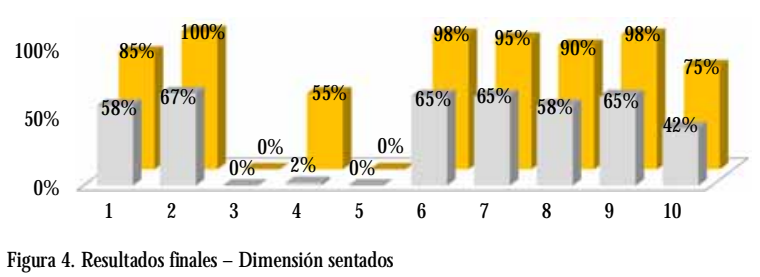

El 80\% -promedio de la población infantil presenta una mejoría en la segunda dimensión del test. Los menores con bajo desarrollo de esta dimensión después de la evaluación post-test son los niños tres y cinco con

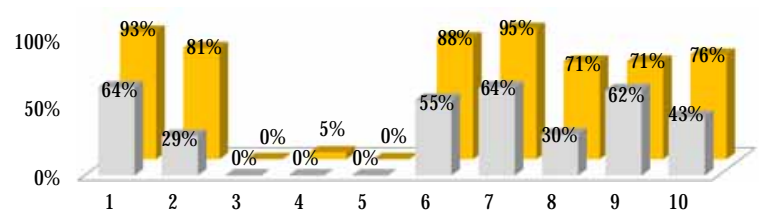

Figura 5. Resultados finales - Dimensión gatas y ar rodillado 
porcentajes de $0 \%$ en ambos casos. Los de mayor porcentaje de desarrollo de esta área son los infantes dos, seis y nueve $\ll 100 \%$, y 98 respectivamente».

El $80 \%$ de la población infantil presenta una mejoría en la tercera dimensión del test. Los menores con bajo desarrollo de esta dimensión después de la evaluación post-test son los menores tres, cuatro y cinco con porcentajes de 0\% para los casos tres y cinco, y de 5\% para el caso cuatro. Los de mayor porcentaje de desarrollo de esta área son los infantes uno, seis, y siete $-93 \%$, $88 \%$ y $95 \%$ respectivamente-

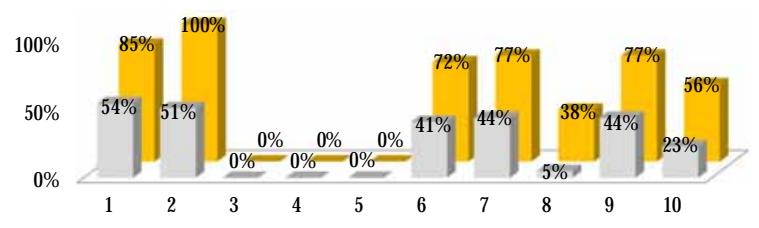

Figura 6. Resultados finales - Dimensión bipedestación.

El 70\% de la población infantil presenta una mejoría en la cuarta dimensión del test. Los analizados con menor desarrollo de esta dimensión después de la evalua ción post-test son los menores tres, cuatro y cinco con porcentajes de $0 \%$ para todos los casos. Los de mayor porcentaje de desarrollo de esta área son los infantes uno, dos, siete y nueve $(85 \%, 100 \%, 77 \%$ paralos casos siete y nueve).

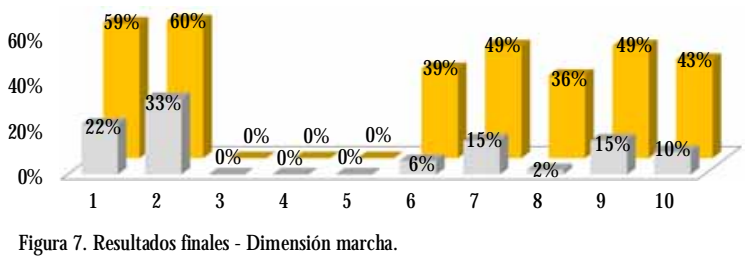

El 70\% de la población infantil presenta una mejoría en la quinta dimensión del test. Los presentados con nulo desarrollo de esta dimensión después de la evaluación post-test son los niños tres, cuatro y cinco con porcentajes de $0 \%$ para todos los casos. Los niños con ma yor porcentaje de desarrollo de esta área son los infan-

Tabla 2.

Resultadosfinales de pre y post test - Escala deAshworth

\begin{tabular}{|c|c|c|c|c|c|c|c|c|c|c|c|c|}
\hline & \multicolumn{12}{|c|}{ Escala deAshworth } \\
\hline & \multicolumn{6}{|c|}{ Pre-test } & \multicolumn{6}{|c|}{ Post-test } \\
\hline & 0 & 1 & $1+$ & 2 & 3 & 4 & 0 & 1 & $1+$ & 2 & 3 & 4 \\
\hline Niño 1 & & $\bar{x}$ & & & & & & $x$ & & & & \\
\hline Niño 2 & & & $\mathrm{x}$ & & & & & & $\mathrm{x}$ & & & \\
\hline Niño 3 & & & & & & $x$ & & & & & & $x$ \\
\hline Niño 4 & & & & & $x$ & & & & & & $x$ & \\
\hline Niño 5 & & & & & $x$ & & & & & $x$ & & \\
\hline Niño 6 & & & $\mathrm{x}$ & & & & & & $x$ & & & \\
\hline Niño 7 & & $\mathrm{x}$ & & & & & & $x$ & & & & \\
\hline Niño 8 & & $x$ & & & & & & $\mathrm{x}$ & & & & \\
\hline Niño 9 & & $x$ & & & & & & $x$ & & & & \\
\hline Niño 10 & & & & & & & & & & & & \\
\hline
\end{tabular}

tes uno, dos, siete y nueve $\ll 56 \%, 60 \%, 49 \%$ para los casos siete y nueve». Resultados finales de pre y post test- Escala de Ashworth.

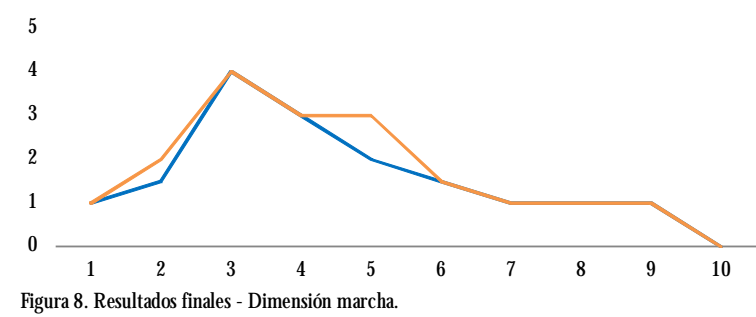

Del $100 \%$ de los evaluados con la Escala de tono muscular deAshworth, el 90\% de la población evaluada no muestra una mejora significativa en cuanto a su desarrollo muscular. El 10\% restante (niño cinco) evidencia una mejora en su tono muscular posterior a la aplica ción de la segunda toma de datos.

\section{Discusión}

Son muchas las conceptual izaciones existentes referentes a la Matrogimnasia y a la PCI sobre todos en niños en edad comprendida de siete años. Sin embargo, dichos conceptos permiten conocer, indagar y enriquecer su funcionalidad para el desarrollo motriz de los menores. Cuando Lucas (2018) se refieren a la Matrogimnasia como método integrador social entre los niños y padres para el mejoramiento cognitivo, ha cen énfasis hacia conocer la M atrogimnasia desde adentro para conocer los beneficios de los ejercicios. A esta postura Fuentes (2013) aclara que realmente si hay que tomar en consideración el significado de la Matrogimnasia, pero al mismo tiempo deben existir las herramientas necesarias para poder llevar a cabo los ejercicios, tales como: colchonetas, implementos para gimnasia de poli motor, música, estimulaciones sensomotoras otros materiales físicos.

En este mismo orden de ideas, Moreno \& Rivas, 0 p. Cit. , exponen que la final idad de la M atrogimnasia es establecer una guía para el desarrollo psicomotriz. El estudio plantea la problemática común en estos casos, como lo es la falta de nuevas estrategias y alternativas de movimiento para lograr la inclusión de los padres de familia dentro del proceso del ámbito psicomotor. Avendaño et al. (2020) señalan que los menores con problemas psicomotores demuestran falencias hacia destrezas motoras gruesas y finas, que delinean la hipótesis de déficit direccionados a retrasos marcados en el desmejoramiento de dominios lingüísticos y motrices. Añaden que la integración entre el lenguaje y las habilidades motoras, no es simple ni adjetiva, sino más bien 
compleja y multifacética. Es por esto que la investigación plantea estudios acerca de la motricidad, tipos de motricidad, psicomotricidad, historia de la Matrogimnasia, beneficios e importancia de la Matrogimnasia.

Al respecto destaca M ejía, O p. Cit. , el cuidado que hay que tener cuando se aplican instrumentos para medir, en este caso, desarrollo motriz en niños con PCl. No es igual medir el estatus del niño con problemas de $\mathrm{PCl}$, que medir lainclusión de los padres para estimular mediante ejercicios que permitan el mejoramiento motriz. Prieto, Galán, Barrero \& Cerro (2020) argumentan que lo motriz es un término extendido considerado como un mecanismo para activar el desarrollo intelectual, psicomotor y personal de los más pequeños. Las mediciones son distantes y los resultados deben ser analizados en conjunto para poder tomar decisiones en pro de mejorar los procesos de adaptación entre procesos conjuntos entre el $\mathrm{PCl}$ y la Matrogimnasia.

Con respecto a la $\mathrm{PCl}$, Q uesada \& Fonseca (2015) expresan que el mismo debe ser visto como como un problema de salud cerebral. Concluyendo que el riesgo de los pacientes con PCI se centra hacia el estado nutricional, es decir, le dan importancia al problema de la desnutrición a través de la valoración global subjetiva. Pero Islas \& C astillejos (2016); Vidal, Vázquez, Morales \& Iturbide (2016) el tratamiento debe ser visto desde varios puntos de vista, tratándose de niños de edades entre cinco y siete años. Al mismo tiempo, se debe tomar en consideración el análisis clínico de la marcha y la postura, presentadas al momento de realizar las respectivas evaluaciones conductual es pertinentes a lo delicado que representa la $\mathrm{PCl}$.

Si laPCI paraDíaz (2016); Cevallos\& M olina(2015) la calidad de vida de estos niños de edad comprendida de siete años, tienen un alto riesgo de desarrollar una calidad de vida regular y mala, debido a la patología presente. Dándole otra visión Yelin (1997) concluye al respecto de la existencia de una asociación directa entre el mal estado nutricional de los pacientes con $\mathrm{PCl}$ y, el desarrollo de una calidad de vida inferior a pacientes con un mejor estado nutricional u otras patologías.

\section{Conclusiones}

Tomando en consideración todos los análisis hermenéuticos, fenomenológicos, descriptivos y, revisando los resultados arrojados al momento de aplicar los instrumentos de la investigación, se puede concluir que la M atrogimnasiaes una herramienta excelente para implementarlas en niños de edad comprendida de siete años con problemas de $\mathrm{PCl}$, debido a las bondades tera péuticas que la misma ofrece.

La participación de los padres y representantes, es esencial durante y después del proceso de la Matrogimnasia, ya que el factor "afecto familiar», juega un papel sumamente importante en el desarrollo cognitivo y motriz del menor. La importancia de informar acerca de esta herramienta por parte de los profesionales que la ponen en práctica, es vital para que las personas responsables, puedan entender el proceso y, apoyar desde el hogar con las diferentes rutinas necesa rias para reforzar las mismas y, obtener resultados satisfactorios en cuanto a tiempo, efectividad y eficiencia.

Cabe destacar que el tiempo de intervención de la Matrogimnasia, mostró resultados alentadores en el desarrollo de habilidades motoras en niños de siete años con PCl. La evidencia de mejoría puede estar determinada por el tiempo, frecuencia y duración de los ejercicios practicados por profesionales tanto del área de salud y educación, el cual se encuentran debidamente especializados en este tipo de métodos. La recomendación principal es profundizar cada una de los ejercicios terapéuticos de practica Matrogimnasia que se realizó en este estudio en un libro, con descripción e instrucciones parasu implementación eficientemente, dado que la mayoría de los padres tuvo bastante dificultad con adaptarse con la orientación de la metodología de implementación.

\section{Limitaciones del estudio}

El estudio es de corte longitudinal, por lo que no se ha podido realizar un seguimiento de la muestra para observar si sus comportamientos son continuados en el tiempo o, algo propio de la edad. Solo han participado niños de siete años de edad, por lo que no se han obtenido unos datos que puedan hacer referencia a toda la etapa pre-adolescentes. En un futuro se podría plantear una investigación incluyendo el grado de dificultad de la $\mathrm{PCl}$, y poder así analizar las diferencias al momento de la implementación de la Matrogimnasia.

\section{Agradecimientos}

Agradezco a Dios por ejercer esta gran profesión de fisioterapista, que me dio la oportunidad de percibir connotaciones relevantes en la U nidad Educativa Especializada Carlos Garbay, durante 20 años con mis niños con discapacidad y los padres de familia, entre ellas, el 
de ser más útil hacia los demás, el entender que la experiencia es el perfil de fortalecimiento en el trabajo de fisioterapia, mediante el effuerzo, dedicación y perseverancia como factores preponderantes en el desa rrollo profesional. Sin lugar a duda, un agradecimiento al MSc. Marco Camacho coordinador de la maestría en Entrenamiento Deportivo, al Dr. O swaldo López director del Departamento de Posgrado y Educación Continua de la U niversidad Estatal de Bolívar, por supuesto al MSc. Diego Bonilla maestro y amigo por sus sabias aportaciones en el proceso de elaboración del Trabajo Final de Master y en el desarrollo científico de este contenido con tinte innovador enfocado en beneficio a la Comunidad Educativa. Estos logros académicos lo dedico con gran gratitud a mi Madre Hilda Vargas y familia. Finalmente, a la Universidad Tecnológica Indoamérica por financiar y apoyar la publicación de este trabajo de investigación.

\section{Referencias}

Alvarado, B., Cervantes, M. ., Carrasco, F., \& García, M. (2015). Parálisis cerebral infantil espásticaen un recién nacido: Presentación de un caso. M edicego, 17 (2), 1-4. Recuperado dehttp:/ / new. medigraphic. com/ cgibin/ resumen.

Anangonó, K. (2016). Estimular el proceso afectivo a través de la matrogimnasia en niños y niñas de 3 años de edad.Video tutorial dirigido a los padresdefamilia del CDI Guagua Centro «Padre Luis Vacari». (Tesisdepregrado).Tecnológico Superior Cordillera, Q uito-Ecuador. Recuperado de https:/ / www. dspace.Cordillera. edu.ec/ bitstream/ 123456789/ 2261/ 1/ 4-DTI-16-16-1725071011.pdf

Avendaño, P., Hernández, C., Fernandes, S., Peña, S., Pavez, G., Troyano, A ..., \& Fernandes, J. (2020). Destrezasmotorasy marcadores Genéticosen niños conTrastorno Específico del lenguajey desarrollo típico del lenguaje. Retos, 38(38), 235241. DO I: https: / / doi.org/ 10.47197/ retos. V38i38.71461

Bonilla, D., Pérez, M. , Jami, P., \& Camacho, M. (2019). Proyectos deestimulación temprana: características esenciales desdela visión metaanalítica. EduSol, 19 (67), 26-38. Recuperado de http:/ / edusol.cug co. cu/ index. php/ EduSol/ article/ view/ $1116 / 2161$

Campistol, J.,Arroyo, H., Póo, P., \& Ruggieri,V. (2011).N Nurología

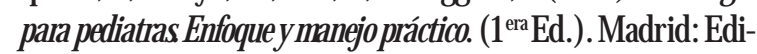
torial MédicaPanamericana.

Cevallos, E., \& Molina, G. (2015). Calidad de vida y necesidades educativas especialesen losniños de 2 a 3 añoscon parál isiscerebral leve. (Tesisdepregrado). Universidad de Guayaquil. Recupera do dehttp:/ / repositorio.ug edu.ec/ bitstream/ redug/ 14663/ 1/ Cevallos\%20-\%20M olina.pdf

Cortés, G. (2003, 08 deagosto). U namiradahistóricaalaevolución de la ciencia. Antroposmoderno. Recuperado de http:/ / antroposmoderno.com/ antro-articulo.Php?id_articulo $=372$
Creswell, J., \& C reswell, JD. (2018). Research design: qualitative, quantitative, and mixed methodsapproaches. (5 $5^{\text {th }} E d$. ). LosAngeles: SAGE.

Eliasson, A., Krumlindem, L., Rösolad, B., Beckung, E., Arner, M., O hrvall, A., \& Rosenbaum, P. (2006). The manual ability classification system for children with cer ebral palsy. M ed Child. Neurol, 48(7), 1-6. Recuperado de http:/ / onlinelibrary.wiley.com/ doi/ 10.1111/ j.14698749. 2006.tb01313.x/ pdf

Fuentes, L. (2013). M trogimnasia: Unnuevo estilo devida. Calameo. Recuperado de https:// es.calameo.com/ read/ $002404203 a b b 7 e d 8 c 0627$

Gómez, S. , Jaimes,V., Palencia, C., Hernández, M ., \& Guerrero, A. (2013). Parálisiscerebral infantil. Venezolanosdepuericultura y pediatría, 76(1), 30-39. Recuperado de https:// www. redalyc.org/ pdf/ 3679/ 367937046008. pdf

Hernández, R., Méndez, S., Mendoza, C., \& Cuevas, A. (2017).

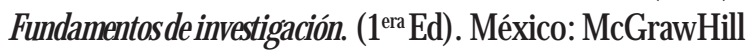
Education.

Hernández-Sampieri, R., \& M endoza, C. (2018). M etodología dela investigación: Las rutas cuantitativa, cualitativa y mixta. (1 era Ed.). México: McGrawHill Education.

Islas, N ., \& Castillejos, M . (2016). Prevalenciade sobrecarga, depresión y nivel dedependenciaen cuidadores primariosinformales deniños con lesión cerebral de un centro de rehabilita ción infantil. Psicologíay Salud, 26(1), 11. Recuperado dehttps:/ / psicologiaysalud.uv.mx/ index. php/ psicysalud/ article/ view/ $1895 / 0$

Jadán, J., Guevara, C., Lara, P., Sánchez, S., Calle, T., Salvador, L... , \& Bonilla, D. (2020). Building hybrid interfacesto increase interaction with young children and children with special needs. In: Nunes I. (eds) Advances in Human factors and systems interaction. AHFE 2019. Advancesin intelligent systemsand Computing, volumen 959. Springer, Cham. DO I: https: / / doi.org/ 10.1007/ 978-3-030-20040-4_28

Jami, L. (2019). La matrogimnasia en el desarrollo delashabilidades motoras gruesas en niños de 7 años con parálisis cerebral infantil en la U nidad Educativa Especial izada Carlos Garbay dela ciudad de Riobamba. (Tesisdepostgrado). Universidad Estatal deBolívar, GuarandaEcuador. Recuperado dehttp:/ / dspace ueb edu.ec/ bitstream/ 123456789/3195/ 1/ MATRO GIMNASIA. pdf

Joaquín, J. (2014). La matrogimnasia: Presenta diversas formas de ejercitación. [M ensaje en un blog]. Recuperado de http:/ / lamatrogimnasia. blogspot.com/

Lieber, E., \&Weisner, T. (2010). M eeting thepractical chall enges of mixed methods research. ( $2^{\text {nd }} \mathrm{Ed}$.). H andbook of mixed methods in social \& behavioral research. Thousand 0 aks, CA: Sage.

López,J. M. ., Moreno, R., \& López, J. L. (2020).Análisisdel impacto deun programadeportivo en niños con trastorno del espectro del autismo. Retos, 39, 98-105. Recuperado dehttps:/ / recyt.fecyt.es/ index. php/ retos/ article/ view/ 74841

López, G. (2015). Matrogimnasia: medio parafortalecer vínculos afectivosentrepadresehijos. ClubEnsayos, 28(1), 1-9. Recupe rado dehttps:/ / www.clubensayos com/ Tema-Variados/ MA TRO GIM NASIA-M EDIO -PARA-FO RTALECER- 
V\%C3\%8DNCULOS-AFECTIVOS/ 2942615. html

Lorente, I. , \& Bugie, C. (1988). Neurología pediátrica:Trastornos motores, parálisiscerebral. BuenosAires, Argentina: El Ateneo Editorial.

Low, N. (1987). Parálisiscerebral: Enfermedadesincapacitantesen el niño. Barcelona, España: Salvat.

Lucas, F. (2018). Utilizar la matrogimnasia para el mejoramiento dela integración social entreniñosy niñasde4 añosdeedad, mediantela elaboración de una guía informativa, dirigido a los padresy madres defamilia del Centro Infantil «San Patricio». (Tesisde pregrado). Tecnológico Superior Cordillera. Recuperado de http:/ / www. dspace. cordillera. edu.ec:8080/ xmlui/ bitstream/ handle/ 123456

789/4131/47-DTI-17-181725132896. pdf? sequence $=1$ \&isAllowed $=y$

Lyon, E. (1990). Patología perinatal: incapacidadesmotricescerebrales. París: N euro-pediatríaM asson.

M ejía, N. (2010). Validación dela escala:Gross motor function measure en niñoscon parálisiscerebral para Colombia. (Tesis de pregrado). UniversidadAutónomadeM anizales. Recuperado de https:/ / repository.ces. edu.co/ bitstream/ 10946/ 2437/ 2/ Gro ss.pdf

Moreno, S., \& Rivas, E. (2017). La importancia dela matrogimnasia en niños de 4-5 años dela U nidad Educativa JoséM ejía Lequerica. (Tesis de pregrado). U niversidad de Guayaquil. Recuperado dehttp:/ / repositorio.ug.edu.ec/ bitstream/ redug/22799 / 1Moreno\%20Rivera\%20\%20Shirley\%20\%20Stefanny $\% 20 y \% 2$ ORivas\%20M osquera\%20\%20Edison\%20Fabricio\%20\%200232017.pdf

Novoa, F. (1991). Parálisis cerebral. (4ªEd.). Santiago de Chile, Chile: Editorial M editerráneo.

O rganización deEstados IberoamericanosparalaEducación, la Cienciay laCultura[O EI]. (2012). Ciencia, tecnología einnovación para el desarrollo y la cohesión social: Programa iberoamericano en la década de losbicentenarios. Madrid, España: 0 El.

Pacheco, J. (2015, 17 de marzo). Alumnosy padresdefamilia practican matrogimnasia. [M ensajeen un blog]. Recuperado dehttp:/ / www.sndigital. mx/ 11929-alumnos-y-padres-de familiarpractican-lamatrogimnasia.html

Pinos, G., Ayala, D. , \& Bonilla, D. (2018). Desarrollo del pensa miento lógico-matemático através dejuegospopularesy tradicionalesen niños deeducación inicial. Revista científica Ciencia y Tecnología, 18(19), 133-141. Recuperado de http:/ / cienciaytecnologia. uteg.edu.ec/ revista/ in dex. php/ cienciaytecnologial article/ view/ 190/ 278

Pinzón, D. (2015). La matrogimnasia y el desarroll o psicomotriz delos niños, educación inicial nivel II; Escuel aVicente Bastidas Reinoso, Loja año 2014. (Tesisdepregrado). Universidad Nacional de Loja, Ecuador. Recuperado dehttp:/ / dspace. unl. edu.ec:9001/ jspui/ bitstream/ 123456789/21118/ TESS\%2OPINZON\%200FICIAL\%2OPRIVADA\%20at\%20dentpdf

Póo, P., \& Campistol, J. (2007). Neuropediatría: parálisiscerebral infantil. Tratado de pediatría. ( 9 na Ed.). Madrid: Editorial $O$ cean/ Ergon.

Prieto, J., Galán, N. , Barrero, D. , \& Cerro, D. (2020). La salade psicomotricidad parael trabajo de educación físicaen educa- ción infantil: un estudio exploratorio. Retos,39, 106-111. DO I: https:/ / doi.org/ 10.47197/ retos. V0i39.78398

Q uesada, L. \& Fonseca, I. (2015). Laparálisiscerebral como un problemadesalud. Correo Científico M édico, 19(4), 757-760. Recuperado dehttps: / / www. medigraphic. com/ cgi-bin/ new/ resumenl. cgi? DARTICULO = 62892

Robaina, G., Riesgo, S. \& Robaina, M. (2013). Definición y clasificación delaparálisiscerebral: ¿Un problemayaresuelto? N eurol, 45(1), 1-8. Recuperado dehttp:/ / www. neurologia.com/ pdf/ Web/ 4502/ y020110. pdf.

Solís, C. (1994). Razoneseintereses: La historia dela ciencia despuésde Kuhn. Barcelona, España: Paidós.

Solórzano, M . \&Vera, H. (2018). Relación entremalnutrición y calidad de vida en pacientes de 4 a 10 años con parálisis cerebral infantil. (Tesisdepregrado). U niver sidad de Guayaquil, Ecua dor. Recuperado dehttp:/ / repositorio.ug edu. ec/ bitstream/ redug/ $34471 / 1 /$ C D \% 2077 - \% 20SO LORZANO \%20SALVATIERRA\%20M ARIA\%20\%2 OKATHERINE\% 3 b\% 20VERA \% 20G O MEZ $\% 2 C \% 20 H E L E N \% 20 M A D E L E Y$.pdf

Strauss, A. \& Corbin, J. (2002). Bases dela investigación cualitativa: Técnicas y procedimientos para desarroll ar la teoría fundamentada. (1 eraEd. ). Colombia: Editorial U niver sidad deAntioquia.

Tatay, A. (2016). Estudio dela densidad ósea en niñosy adol escentescon afectación moderada y severa por parálisiscerebral. (Tesis doctoral). U niversidad deSevilla, España. Recuperado dehttps:/ / idus. us. es/ bitstream/ handle/ 1441/ 39821/ TESIS\%20D O C\%20DEF\%20v.2.pdf?sequence=1\&isAllowed=y

U ribe, B. (2017). La historiade la ciencia: ¿Q ué es y paraqué? Odontológica Mexicana, 21(2), 78-80. Recuperado de http:/ / www. elsevier.es/ es-revistarevista-odontologica-mexicana 90-pdf-S1870199X17300277.

Vázquez, C., \&Vidal, C. (2014). Parálisiscerebral infantil: D efinición y clasificación através delahistoria. Mexicana deO rtopedia Pediátrica, 16(1), 6-10. Recuperado de https:// www. medigraphic. com/ pdfs/ opediatria/ op-2014/ op141b.pdf

Vidal, C., Vázquez, C., M orales, M . \& Iturbide, P. (2016).Tratamiento en pacientes con parálisiscer ebral infantil de acuerdo con el análisis clínico de la marchay la postura. M exicana de Ortopedia Pediátrica, 18(1), 46-50. Recuperado de https: / / www. medigraphic. com/ pdfs/ opedia tria/ op-2016/ op161h.pdf

Wollack J. (2004). Encefalopatías estáticas. Madrid, España: McG rawHill Interamericana.

Yelin, B. (1997). Diagnóstico temprano dela parálisiscerebral. Neurol, 25(141), 725-727. Recuperado dehttp:/ / www.sd.cu/ galerias/ pdf/ sitios/ rehabilitacion-temprana/ diagnostico_ temprano_de_la_pc_1_2.pdf 